\title{
Estudios de parentesco mediante marcadores del ADN : Experiencia en resolución de casos en los últimos seis años
}

\author{
Hugo Jorquera G ${ }^{1 a}$, Mónica Acuña P2a, Lucía Cifuentes L². \\ Kinship determination \\ using DNA markers
}

Background: Autosomal and Y chromosome short tandem repeats (STRs) and mitochondrial DNA polymorphisms are the most commonly used molecular tools for determination of kinship. Aim: To report a revision of 1,120 kinship cases (paternity and others) analyzed in our laboratory. Material and methods: Revision of all kinship cases analyzed between years 2001-2006. Autosomal and Y chromosome STRs and mitochondrial DNA polymorphisms were analyzed in DNA extracted from blood samples. Results: Paternity was excluded in $27.2 \%$ of cases. This figure did not change significantly along years. Most paternity exclusions were confirmed by the discordance in 5 genetic markers (30.5\%), followed by exclusion of 4 and 6 genetic markers (20.3 and 20\% respectively). Two studied cases were paternal and maternal exclusions, corresponding to a change of children between two families. In one case, the paternal line was assessed through Y chromosome markers, studying 16 STRs of this chromosome, positively confirming this paternal relationship. Another case was analyzed for maternal line using mitochondrial DNA analysis. In six cases, a genetic marker with a paternal-sibling mutation, was observed. The criteria for the determination of mutation was the finding of only one discordant marker between at least thirteen markers analyzed in each case. Also, an increase or decrease in one unit repeated in tandem (tetranucleotide) between the alleged father and the son was also required. One subject had a double mutation. In this case, paternity was confirmed analyzing thirteen autosomic STRs and five Y-STRs. Conclusions: The authors have acquired great expertise in kinship analysis and had established criteria to solve complex kinship cases (Rev Méd Chile 2008; 136: 193-200).

(Key words: DNA; Chromosomes; Family; Paternity)

\footnotetext{
Recibido el 8 de mayo, 2007. Aceptado el 10 de septiembre, 2007.

${ }^{1}$ Genética y Tecnología Ltda. ${ }^{2}$ Programa de Genética Humana, Instituto de Ciencias Biomédicas (ICBM), Facultad de Medicina, Universidad de Chile. Santiago de Chile.

aTecnólogo Médico, Magíster en Ciencias Biológicas
}

L a mayor parte de los casos de investigación de parentesco están relacionados con la determinación de la paternidad biológica, sin embargo, se dan muchos casos en que es necesario abordar

Correspondencia a: Hugo Jorquera González. Genética y Tecnología Ltda. Coronel Santiago Bueras 160-A, Santiago. Fono: 63316 19. Fax: 63931 24. E mail: hjorquera@genytec.cl otros desafíos, como son los que conciernen a estudios de paternidad/maternidad indirectos (abuelidad), hermandad o pertenencia a una determinada línea de parentesco paterna o materna.

Hoy en día, el arsenal de marcadores moleculares del ADN disponibles para tales efectos, permite resolver satisfactoriamente la mayoría de estos casos, sin embargo, las posibilidades de obtener resultados 
con un alto nivel de confianza no sólo dependen de las herramientas técnicas empleadas, sino también de la complejidad del caso y, especialmente, de la disponibilidad de los familiares de referencia.

Existen numerosas herramientas moleculares disponibles para enfrentar este tipo de casos. Entre ellas se cuentan el uso de marcadores STRs autosómicos (short tandem repeats), STRs del cromosoma Y y polimorfismos del ADN mitocondrial a nivel de la región control. Los primeros resultan altamente informativos para la determinación de paternidad y maternidad, ya que son heredados mendelianamente en forma codominante. Los STRs del cromosoma Y permiten establecer relaciones patrilineales, por cuanto este cromosoma se hereda en bloque y casi sin recombinación (haplotipo) desde los padres a los hijos varones. Por último, los polimorfismos del $\mathrm{ADN}$ mitocondrial permiten realizar relaciones matrilineales, ya que este genoma es transmitido en forma completa (haplotipo) sin recombinación, desde la madre a todos sus hijos, pero es heredado únicamente por línea matema. El uso de cada uno de ellos por separado o en combinación, puede resolver la mayoría de los casos de consultas por parentesco de manera suficientemente segura.

Desde el punto de vista genético, existen varios índices que permiten valorar estadísticamente los resultados obtenidos en las pruebas de parentesco. Los más comunes son el índice de paternidad o parentesco (L) o likelihood ratio (LR) y la probabilidad de paternidad o parentesco ${ }^{1,2}$.

Ambos indicadores están relacionados entre sí y valoran la probabilidad de que el supuesto padre lo sea en realidad, considerando los alelos que el hijo recibe de su progenitor y las frecuencias génicas con que dichos alelos están representados en la población. En nuestro país se acepta como mínimo una probabilidad de paternidad (L) de 99,9\% para considerar legalmente la paternidad "acreditada"3, lo cual es ligeramente mayor que los que se exige en varios países de Europa, en donde la paternidad es probada al alcanzar un L de 99,7\% 4 .

Los criterios de exclusión más aceptados en la actualidad, comesponden a la discordancia en al menos tres o más marcadores genéticos entre el padre presunto y el hijo estudiado, esto, porque existe la probabilidad de que una discordancia sea producto de una mutación y no de una situación de exclusión ${ }^{5}$.

Este criterio se ha ido ampliando a medida que los sistemas de análisis han aumentado el número de loci estudiados en cada caso. Es así como hace unos años bastaban dos marcadores discordantes para considerar una paternidad excluida 6 . Sin embargo, al aumentar el número de loci analizados, también aumenta la probabilidad de hallar mutaciones padre/hijo, debido a que el polimorfismo de estos marcadores genéticos es generado, en parte, por la alta tasa de mutación que presentan éstos, razones que han llevado a los autores a proponer otros criterios para el cálculo de la probabilidad de exclusión?.

El objetivo del presente trabajo es mostrar la experiencia de 6 años en resolución de casos de paternidad y otros casos de parentesco atípicos, realizados sobre casos privados (no judiciales) en nuestro país.

\section{MATERIAL Y MÉTODOS}

El trabajo presentado muestra un resumen de 1.120 casos de determinación de parentesco llevados a cabo en el laboratorio de Genética y Tecnología Ltda. desde el año 2001 a 2006. La mayor parte de los casos correspondió a determinación de la paternidad biológica, aunque se pudieron analizar una serie de otras consultas tales como determinación de abuelidad paterna, determinación de pertenencia a una determinada línea paterna, determinación de hermandad matema, determinación de patemidad prenatal y determinación simultánea de patemidad/ maternidad en dos casos con sospecha de cambio de neonatos en un hospital público.

Tipificación de marcadores STRs autosómicos y del cromosoma Y. El ADN fue extraído desde muestras de sangre mediante un método orgánico estándar utilizando fenol/cloroformo y precipitación con etanol. El ADN obtenido fue cuantificado espectrofotométricamente utilizando un instrumento GeneQuant (Amersham).

Para la tipificación de los STRs autosómicos se utilizaron los sistemas AmpFeSTR Profiler Plus ${ }^{\mathrm{TM}}$, AmpFeSTR Cofiler ${ }^{\mathrm{TM}}$ (Applied Biosystems) y Poweplex16 $6^{\mathrm{TM}}$ (Promega Corp). Los STRs del cromosoma Y fueron tipificados mediante el uso de los sistemas AmpFeSTR $^{\circledR}$ Yfiler $^{\mathrm{TM}}$ (Applied Biosystems) e Y-PLEX $5^{\mathrm{TM}}$ (ReliaGene Technologies).

Entre 0,2-5 ng de ADN fue utilizado para ser amplificado mediante PCR en un termociclador Applied Biosystems modelo GeneAmp 9700. Los 
productos amplificados fueron separados electroforéticamente, utilizando un analizador genético automático de fluorescencia inducida por láser ABI-310. Los genotipos resultantes determinados automáticamente mediante el programa Genotyper V 2.5

Tipificación de la región control del ADN mitocondrial. El análisis de la región control del ADN mitocondrial se realizó mediante el protocolo desarrollado por la Unidad de ADN II del Federal Bureau of Investigation (FBI) de los Estados Unidos de Norteamérica. Para ello se realizó la amplificación del ADN mitocondrial con los partidores desarrollados para la regiones hipervariables I y II (HVI-HVII $^{8}$. Los productos amplificados fueron purificados mediante columnas de filtración Microcon-100 (Millipore) y luego se determinó su concentración a través electroforesis en geles de agarosa al $1 \%$, teñidos con bromuro de etidio utilizando como referencia un estándar de masa de ADN.

Los productos de las regiones HVI y HVII fueron secuenciados por secuenciación cíclica mediante el sistema BigDye V 1.1 (Applied Biosyste$\mathrm{ms})$. Los productos de secuenciación fueron luego purificados por precipitación con etanol al $75 \%$.

Finalmente, los productos fueron analizados en un secuenciador automático modelo ABI-310 (Applied Biosystems) y los electroferogramas editados mediante el programa Sequence Analysis V 3.4.1.

Las secuencias obtenidas fueron comparadas con la secuencia de referencia de Cambridge revisada (rCRS) ${ }^{9}$ y los polimorfismos señalados como variaciones respecto de dicha secuencia.

\section{Resultados}

Estudios de determinación de paternidad biológica. La Tabla 1 muestra el total de casos de investiga- ción de la paternidad biológica llevados a cabo en nuestro laboratorio entre los años 2001 y 2006. Del total de casos analizados (1.120), se observó que $72,77 \%$ de los casos resultaron en la confirmación de la paternidad biológica y $27,23 \%$ de ellos correspondió a exclusión de la paternidad.

Si observamos los resultados porcentuales parciales por año, vemos que la situación en relación a los casos excluidos se mantiene relativamente constante, con un promedio de 27,24\%. Este valor resulta similar a otros países como Estados Unidos de Norteamérica en el cual, según el reporte de 2004 de la American Association of Blood Banks (AABB), informa la existencia de $25,92 \%$ de casos de exclusión paterna en laboratorios acreditados por dicha institución ${ }^{5}$.

Este resultado contrasta con los porcentajes de exclusión promedio obtenidos durante la década 1990-99 en los casos investigados por el Servicio Médico Legal, en los cuales la tasa de exclusión era cercana a $17 \%$, aun cuando ambos universos no son comparables ya que este último sólo investiga casos judiciales que la mayoría de las veces son iniciados por demanda de la madre.

Número de marcadores genéticos que confirman la exclusión. Los resultados indicados en la Tabla 2 muestran aquellos casos de exclusión de la paternidad en relación al número de marcadores genéticos que confirman dicho resultado.

Cabe señalar que según los criterios internacionalmente aceptados, un resultado de exclusión no puede ser afirmado si existe exclusión entre padre e hijo en sólo un locus. Debido a las altas tasas de mutación mostradas por algunos loci STR utilizados en este tipo de estudios, la presencia de una única discordancia entre padre e hijo, sería atribuible a este fenómeno y no puede utilizarse como criterio de exclusión per se.

Tabla 1. Casos estudiados entre años 2001-2006, según resultados de atribución/exclusión de la paternidad

\begin{tabular}{|lrrrrrrrrrrrr|r|}
\hline & \multicolumn{1}{c}{$2001-2002$} & \multicolumn{1}{c}{2003} & \multicolumn{1}{c}{ Peńodo } & \multicolumn{1}{c|}{ 2004 } & \multicolumn{2}{c|}{ Totales } \\
Resultados & $\mathrm{N}$ & $\%$ & $\mathrm{~N}$ & $\%$ & $\mathrm{~N}$ & $\%$ & $\mathrm{~N}$ & $\%$ & $\mathrm{~N}$ & $\%$ & $\mathrm{~N}$ & $\%$ \\
\hline Inclusión de la paternidad & 147 & 71,0 & 154 & 76,6 & 135 & 70,3 & 200 & 72,5 & 179 & 73,4 & 815 & 72,77 \\
Exclusión de la paternidad & 60 & 29,0 & 47 & 23,4 & 57 & 29,7 & 76 & 27,5 & 65 & 26,6 & 305 & 27,23 \\
Total & 207 & 100,0 & 201 & 100,0 & 192 & 100,0 & 276 & 100,0 & 244 & 100,0 & 1.120 & 100 \\
\hline
\end{tabular}


Tabla 2. Casos de exclusión de la paternidad y número de marcadores que confirmaron dicho resultado

\begin{tabular}{|crrrrrrr|r|}
\hline $\begin{array}{l}\text { No marcadores } \\
\text { que excluyen }\end{array}$ & $2001-2002$ & 2003 & 2004 & $\begin{array}{r}\text { Período } \\
2005\end{array}$ & 2006 & $\mathrm{~N}$ & $\%$ \\
\hline 3 & 2 & 5 & 1 & 5 & 8 & 21 & 6,9 \\
4 & 11 & 12 & 10 & 16 & 13 & 62 & 20,3 \\
5 & 14 & 16 & 23 & 26 & 14 & 93 & 30,5 \\
6 & 12 & 6 & 8 & 12 & 23 & 61 & 20,0 \\
7 & 14 & 3 & 7 & 12 & 8 & 44 & 14,4 \\
8 & 4 & 2 & 5 & 1 & 3 & 15 & 4,9 \\
9 & 3 & 1 & 1 & 2 & & 7 & 2,3 \\
10 & 60 & 45 & 57 & 74 & 69 & 305 & 100,0 \\
\hline
\end{tabular}

La Tabla 2 muestra que en la mayoría de los casos (30,5\%), la exclusión se llevó a cabo con 5 marcadores discordantes entre padre e hijo, y $70 \%$ de ellos fue confirmado entre 4 y 6 marcadores discordantes (216 casos sobre 305 exclusiones).

Índice de paternidad combinado. Los casos de paternidad estudiados durante el período analizado fueron realizados sobre la base de al menos nueve STRs autosómicos de herencia independiente, llegando a analizar hasta dieciséis STRs independientes cuando el caso así lo requería. El índice de paternidad combinado utilizado como límite inferior para informar los casos de atribución de la paternidad fue de 1.000, lo cual está en concordancia a los requerimientos dictados por el Servicio Médico Legal en su Normativa Técnica, la que exige una probabilidad mínima de 99,9\% para la acreditación de la paternidad biológica.
Casos de paternidad atribuida con mutación paterna. Del total de casos estudiados, en 10 de ellos se encontró sólo un alelo discordante entre el padre y el hijo, lo que se interpretó como mutación. En todos ellos se realizó confirmación de la paternidad con el mayor número de marcadores genéticos disponibles ${ }^{10,11}$ para asegurar la presencia de sólo un locus discordante.

En todos los casos la diferencia observada entre el padre y el hijo para el alelo considerado como mutado, fue de sólo un motivo repetido (tetranucleótido), lo cual es utilizado como criterio adicional para la determinación de efecto mutacional.

La Tabla 3 muestra las tasas de mutación calculadas a partir de los 1.120 casos estudiados, en comparación con las tasas de mutación publicadas en el extranjero $^{12}$. Nuestras tasas no fueron estadísticamente distintas de las extranjeras, excepto para el locus FGA, en la cual encontramos

Tabla 3. Tasas de mutación calculadas en el estudio y comparación con datos del extranjero

\begin{tabular}{|lcc|}
\hline Locus & Actual estudio & Tasas mutación (\%) \\
\hline VWA & 0,1339 & Extranjero (6) \\
D18S51 & 0,0446 & 0,16 \\
D7S820 & 0,0446 & 0,25 \\
D21S11 & 0,1339 & 0,10 \\
D8S1179 & 0,0446 & 0,21 \\
FGA & 0,0446 & 0,13 \\
\hline
\end{tabular}


una tasa mucho más baja, sin embargo, esta conclusión es aún muy preliminar ya que nuestras estimaciones han sido hechas en base a una muestra muy pequeña en comparación con los estudios extranjeros, por lo que el error estándar de nuestros valores es muy grande. Dado a que estos mismos estudios señalan tasas para los loci FGA, D18S51 y D21S11 de 0,16\%, 0,25\% y 0,21\%, respectivamente, la postura adoptada por nuestro laboratorio es la de excluir solamente cuando en un caso se observan 3 o más loci discordantes entre padre e hijo. Del total de casos analizados, ninguno mostró discordancia entre la madre y el hijo atribuible a mutación.

Sólo un caso estudiado reveló una doble mutación, la cual fue confirmada mediante el estudio de trece marcadores STR autosómicos más 6 STR del cromosoma Y (Tabla 4).

Estudio de línea paterna.Una consulta investigada correspondió a la determinación por parte del interesado de su pertenencia a una determinada familia por vía paterna. Su supuesto padre biológico había fallecido y sólo se contaba con un supuesto tío paterno y un supuesto medio hermano paterno. Para la determinación de este caso se recurrió a un análisis de STRs del cromosoma Y. Se analizó un total de 16 STRs del cromosoma Y, los cuales mostraron un perfil coincidente en las tres personas analizadas (Tabla 5).

Para la determinación de la frecuencia del haplotipo hallado se consultó varias bases de datos de población caucásica, sin encontrarse en ninguna de ellas el perfil estudiado. El resultado se elaboró en base al método de recuento, indicando que resultó en una secuencia única hallada entre 1.276 haplotipos consultados.

Estudio de línea materna. Un caso consultado requería la investigación de hermandad entre dos personas de distinto sexo. En este caso no existían los padres (ya fallecidos) ni tampoco otros hermanos. La única alternativa fue la investigación de línea materna mediante análisis de ADN mitocondrial.

Se analizó las regiones HV1 y HV2 conformándose el haplotipo mostrado en la Tabla 6. Ambas personas mostraron idéntico haplotipo, por lo que pudo realizarse un cálculo de pertenencia a la misma línea materna, que resultó de 1 en 1.056, con un límite superior de confianza de 95\% =1/356.

Estudios de paternidad prenatales. Una línea de investigación de paternidad prenatal se ha seguido a raíz de una creciente demanda por este tipo de casos desde centros gíneco-obstétricos.

Tabla 4. C aso de doble mutación paterno-filial analizada con 13 ST R autosómicos y cinco ST Rs del cromosoma $Y$

\begin{tabular}{|lccccccc|}
\hline LOCUS & D3S1358 & vWA & FGA & AMEL & D8S1179 & D21S11 & D18S51 \\
\cline { 2 - 8 } Padre presunto & $16 / 16$ & $17 / 17$ & $21 / 23$ & X/Y & $13 / 15$ & $30 / 33.2$ & $14 / 14$ \\
Hijo & $16 / 18$ & $16 / 17$ & $23 / 26$ & X/Y & $14 / 15$ & $32.2 / 32.2$ & $14 / 18$ \\
Madre & $17 / 18$ & $16 / 16$ & $20 / 26$ & X/X & $8 / 15$ & $32.2 / 33.2$ & $14 / 18$ \\
& D5S818 & D13S317 & D7S820 & D16S539 & TH01 & TPOX & CSF1PO \\
Padre presunto & $9 / 13$ & $11 / 12$ & $10 / 10$ & $12 / 12$ & $9.3 / 9.3$ & $8 / 11$ & $10 / 12$ \\
Hijo & $7 / 9$ & $12 / 12$ & $10 / 10$ & $10 / 12$ & $7 / 9.3$ & $11 / 11$ & $12 / 12$ \\
Madre & $7 / 9$ & $11 / 12$ & $10 / 11$ & $9 / 10$ & $6 / 7$ & $8 / 11$ & $9 / 12$ \\
& & & & & \\
Padre presunto & DYS393 & DYS19 & DYS389II & DYS390 & DYS391 & & \\
Hijo & 13 & 14 & 29 & 24 & 11 & & \\
\hline
\end{tabular}

En gris se señalan los loci que presentan mutación. 
Tabla 5. Estudio de línea paterna en base a 16 ST Rs del cromosoma Y

\begin{tabular}{|lccccccccc|}
\hline & DYS391 & DYS389 & DYS439 & DYS389II & DYS438 & DYS437 & DYS19 & DYS392 & DYS393 \\
\cline { 2 - 10 } Hijo & 10 & 14 & 12 & 31 & 12 & 15 & 14 & 13 & 13 \\
Tío paterno & 10 & 14 & 12 & 31 & 12 & 15 & 14 & 13 & 13 \\
Hermano paterno & 10 & 14 & 12 & 31 & 12 & 15 & 14 & 13 & 13 \\
& \multicolumn{2}{c}{ DYS390 } & DYS385 & DYS456 & DYS458 & DYS635 Y & GATA H4 DYS448 & \\
Hijo & 24 & 11 & 14 & 16 & 17 & 23 & 12 & 19 & \\
Tío paterno & 24 & 11 & 14 & 16 & 17 & 23 & 12 & 19 \\
Hermano paterno & 24 & 11 & 14 & 16 & 17 & 23 & 12 & 19 \\
\hline
\end{tabular}

Tabla 6. Estudio de línea materna en base a secuenciación de la Región C ontrol del mtAD N

\begin{tabular}{|c|c|c|c|c|c|}
\hline \multirow{3}{*}{$\begin{array}{l}\text { Hermana presunta } \\
\text { Hermano presunto }\end{array}$} & \multicolumn{5}{|c|}{ HV1 } \\
\hline & $16187 \mathrm{~T}$ & $16223 \mathrm{~T}$ & $16290 \mathrm{~T}$ & $16325 \mathrm{C}$ & $16362 \mathrm{C}$ \\
\hline & $16187 \mathrm{~T}$ & $16223 \mathrm{~T}$ & $16290 \mathrm{~T}$ & $16325 \mathrm{C}$ & $16362 \mathrm{C}$ \\
\hline & \multicolumn{5}{|c|}{ HV2 } \\
\hline Hermana presunta & 73G & $204 C$ & $245 \mathrm{C}$ & 263G & $315.1 \mathrm{C}$ \\
\hline Hermano presunto & 73G & $204 C$ & $245 \mathrm{C}$ & 263G & $315.1 \mathrm{C}$ \\
\hline
\end{tabular}

En todos los casos la muestra fue tomada por un especialista ginecólogo-obstetra con amplia experiencia en la toma de muestras de vellosidad coriónica y líquido amniótico, quien derivó la muestra a nuestro laboratorio.

La extracción de ADN desde células fetales en ambos tipos de muestra ha sido muy eficiente y con un éxito de $100 \%$ en la obtención de un perfil genético único del feto, sin contaminación con ADN materno.

Estudios de abuelidad. Durante el período descrito se realizaron tres estudios de determinación de abuelidad paterna. En todos los casos se requería la investigación de la paternidad estando el padre presunto fallecido. De los tres casos estudiados, uno resultó excluido y dos resultaron en atribución positiva de la abuelidad. En todos los casos se analizó un total de trece STR autosómicos, resultando uno de ellos con un índice de abueli- dad combinado de 3.388 (PA 99,97\%) y el otro de 67.655 (PA 99,998\%)

Casos atípicos. Dos casos se podrían considerar atípicos y vale la pena mencionarlos por ser poco frecuentes en la práctica de rutina.

Un caso analizado correspondió a la determinación de la paternidad entre dos padres presuntos que además eran hermanos y mellizos. El estudio demostró que ambos eran gemelos monozigóticos por lo que el caso no se pudo resolver.

Un segundo caso correspondió al estudio de dos casos en que se sospechaba el cambio de dos recién nacidos durante el parto, situación que se investigó mediante $\mathrm{ADN}$ a casi un año de ocurido el nacimiento. Los resultados demostraron que efectivamente dichos niños habían sido identificados erróneamente y entregados a las familias equivocadas, ya que en ambos casos se confirmó la exclusión paterna como matema. Al realizar el intercambio 
entre las dos familias, la inclusión fue categórica por lo que el hecho quedó objetivamente constatado.

\section{DisCUSIÓN}

Los desafíos que hoy en día representa la resolución de casos de parentesco se han ampliado en la medida que las tecnologías disponibles y el conocimeinto de nuevos marcadores genéticos han ido emergiendo, tanto de la investigación básica como de la instrumentación. En nuestro país, a principios de la década 1990-99, los autores del presente trabajo comenzaron con la introducción de técnicas moleculares en la resolución de casos de paternidad con la utilización de dos variantes técnicas: El ADN fingerprinting con sondas multilocus y por otra parte, marcadores VNTRs amplificados por PCR como los loci D1S80 y APO B. Sin embargo con el paso de los años, estos marcadores genéticos, si bien informativos en ese momento, fueron siendo reemplazados por técnicas de mayor facilidad técnica y en las cuales la interpretación subjetiva de los resultados fue reduciéndose. Al respecto, cabe destacar que la introducción en nuestro país a finales de la década 1990-99 de los analizadores genéticos automatizados (conocidos mayoritariamente como secuenciadores de ADN) representó un hito tecnológico en esta área debido a que la presición, sensibilidad y reproducibilidad de los resultados obtenidos mediante esas plataformas garantiza análisis de gran robustez. La introducción del estudio de STRs para el coromosma Y y el análisis de polimorfismos del ADN mitocondrial vinieron aparejados con el desarrollo tecnológico que permitió introducir nuevos marcadores genéticos al arsenal de herramientas para el estudio de parentescos.

Respecto de los casos de paternidad estudiados, el porcentaje de casos excluidos se ha mantenido casi sin variaciones durante los últimos seis años y estos valores son similares a los obtenidos por laboratorios privados de Estados Unidos de Norteamérica consultados por la $\mathrm{AABB}^{5}$. Por otra parte, dado el número de marcadores genéticos utilizados en la actualidad, que puede llegar a 16 en un estudio, nos parece prudente establecer como límite inferior la consideración de a lo menos tres marcadores genéticos discordantes entre el padre y el hijo para excluir una paternidad. La incorporación de un mayor número de marcadores genéticos para análisis de paternidad (Sistemas Identifyler, Applied Biosystems y Powerplex 16, Promega Corp) con un panel de 16 STRs de detección simultánea, lleva implícita una mayor probabilidad de detectar mayor número de mutaciones, por lo que los criterios de exclusión deben ajustarse a esta nueva reali$\operatorname{dad}^{7,10,11}$.

Respecto del establecimiento de valores referenciales para la determinación positiva de la paternidad biológica, este tema fue abordado por la Normativa Técnica desarrollada por el Servicio Médico Legal ${ }^{3}$ en la cual, tras consultar a varios expertos en el tema, se concordó establecer que para que una prueba de filiación sea considerada "legalmente válida" en Chile, en primer lugar el sistema o grupo de marcadores genéticos utilizados debe tener un poder de exclusión a priori mínimo de 0,999. Por otra parte, en el mismo documento se establece que una probabilidad de paternidad a posteriori igual o superior a $99,9 \%$ dará cuenta de una "paternidad biológica acreditada". Estos criterios de valoración de la prueba son ligeramente más exigentes que los aplicados en algunos países europeos, en los cuales se utiliza como umbral de certeza 99,7\%4.

La incorporación de otros marcadores genéticos no autosómicos como STRs del cromosma $\mathrm{Y}^{13} \mathrm{y}$ polimorfismos del mtDNA ${ }^{14,15}$ permiten al laboratorio la investigación de casos más complejos, en los cuales los STRs autosómicos presentan limitaciones. Una de ellas es la determinación de la hermandad en aquellos casos en los cuales no se dispone de los padres. Para esos efectos, en el caso de hermanos varones, la investigación de STRs del cromosoma Y puede aportar importantes antecedentes a la resolución de estos casos por cuanto existen kits con paneles de hasta 16 STRs (Yfiler ${ }^{\circledR}$, Applied Biosystems). También queda demostrada la utilidad del estudio de polimorfismos del mtADN especialmente en casos en que las personas analizadas son mujeres. Los estudios de abuelidad realizados demuestran que en presencia de ambos abuelos se pueden lograr índices de paternidad combinados superiores a 1.000, como lo demuestran dos casos en los cuales la abuelidad fue atribuida con 99,97 y 99,998\%. La posibilidad de realizar un alto número de casos enfrenta a los 
especialistas con situaciones atípicas como las descritas (padres presuntos gemelos, exclusión materna/paterna) que si se desconocen los antecedentes del caso en particular, pudieran inducir a pensar que los resultados obtenidos son producto de un error de laboratorio y no a una situación real.

La demanda creciente por estudios de paternidad que ha tenido lugar en nuestro país en los últimos años, ha permitido que la población conozca cada vez más las posibilidades que brindan los exámenes genéticos en el estudio de parentescos. Es por ello que cada vez con más frecuencia se realizan consultas no sólo referentes

\section{REFERENCIAS}

1. Majumder PP, Nei M. A note on positive identification of paternity by using genetic markers. Hum Hered 1983; 33: 29-35.

2. Cifuentes L, Castilo S, Armanet L, Llop E, Cruz Соке R. Uso de marcadores genéticos en pruebas de paternidad. Rev Méd Chile 1990: 118: 942-3.

3. Instrucciones y normativa técnica sobre pruebas biológicas para la determinación de la paternidad y/o maternidad. Resolución № 1.450 exenta Servicio Médico Legal. Santiago, 29 de diciembre de 1999.

4. LoRente, JA, Lorente M. En: El ADNy la identificación en la Investigación Criminal y en la Paternidad Biológica. 1995, Editorial Comares, Granada, España.

5. The American Association of Blood Banks. Annual Report Summary 2004.

6. Jorquera $\mathrm{H}$, Acuña $\mathrm{M}$, Cifuentes L Análisis de paternidad utilizando cuatro marcadores de ADN amplificados mediante la reacción en cadena de polimerasa. Rev Méd Chile 1999; 127: 1057-62.

7. Cifuentes L, Martínez E, Acuña M, Jorquera $H$. Probability of Exclusion in Paternity Testing: Time to Reassess. J Forensic Sci 2006; 51: 349-50.

8. Wiuson MR, Polanskey D, Buttier J, DiZinno JA, Reploge J, Budowle B. Extraction, PCR amplification and sequencing of mitochondrial DNA from human hair shafts. BioTechniques 1995; 18: 662-9. a la investigación de la patemidad, sino a la determinación de otras líneas de parentesco como son el vínculo entre presuntos hermanos o bien pertenencia a una determinada línea paterna 0 materna. Esto representa un desafío desde el punto de vista genético, ya que exige al laboratorio contar con el mayor número de marcadores genéticos posibles y no limitados solamente a los STRs autosómicos ya que como se ha visto, en muchos casos la información otrogada por los STR del cromosoma $Y$ y los marcadores extranucleares como los polimorfismos del ADN mitocondrial resultan de vital utilidad.

9. Andrews RM, Kubacka I, ChinNery PF, Lightowiers RN, TuRNBull DM, Howell N. Reanalysis and revision of the Cambridge reference sequence for human mitochondrial DNA. Nature Genetics 1999; 23: 147.

10. Krenke BE, Tereba A, Anderson SJ, Buel E, Culhane S, FinIS CJ ET AL. Validation of a 16-locus fluorescent multiplex system. J Forensic Sci 2002; 47: 773-85.

11. Holt CL, Buoncristiani M, Wawin JM, Nguyen T, LAZARUK KD, WALSH PS. TWGDAM validation of AmpFISTR PCR amplification kits for forensic DNA casework. J Forensic Sci 2002; 47: 66-96.

12. ButTLER J. En: Short Tandem Repeat DNA Internet Database: Mutation Rates for Common Loci, disponible en http://www.cstl.nist.gov/biotech/ strbase/mutation.htm (Consultado el 21 de enero de 2007).

13. Santos FR, Pena SD, Epplen JT. Genetic and population study of a Y-linked tetranucleotide repeat DNA polymorphism with a simple nonisotopic technique. Hum Genet 1993; 90: 655-6.

14. Anderson S, Bankier AT, Barrell BG, de Brujun MH, Coulson AR, Drouin J et al. Sequence and organization of the human mitochondrial genome. Nature 1981; 290: 457-65.

15. Wilson MR, DiZinno JA, Polanskey D, Replogie J, Budowle B. Validation of mitochondrial DNA sequencing for forensic casework analysis. Int J Legal Med 1995; 108: 68-74. 